\title{
Polymer Nanofibers via Nozzle-Free Centrifugal Spinning
}

\author{
R. T. Weitz, ${ }^{*} \dagger$ L. Harnau, ${ }^{\ddagger}, \S$ S. Rauschenbach, ${ }^{\dagger}$ M. Burghard, ${ }^{\dagger}$ and K. Kern ${ }^{\dagger}, \|$ \\ Max Planck Institute for Solid State Research, Heisenbergstr. 1, 70569 Stuttgart, \\ Germany, Max Planck Institute for Metals Research, Heisenbergstr. 3, 70569 Stuttgart, \\ Germany, Institute for Theoretical and Applied Physics, Universität Stuttgart, \\ Pfaffenwaldring 57, 70569 Stuttgart, Germany, and Institut de Physique des \\ Nanostructures, Ecole Polytechnique Fédérale de Lausanne, 1015 Lausanne, \\ Switzerland
}

Received January 14, 2008

\begin{abstract}
We report on the unexpected finding of nanoscale fibers with a diameter down to $25 \mathrm{~nm}$ that emerge from a polymer solution during a standard spin-coating process. The fiber formation relies upon the Raleigh-Taylor instability of the spin-coated liquid film that arises due to a competition of the centrifugal force and the Laplace force induced by the surface curvature. This procedure offers an attractive alternative to electrospinning for the efficient, simple, and nozzle-free fabrication of nanoscale fibers from a variety of polymer solutions.
\end{abstract}

Polymer nanofibers are attractive building blocks for functional nanoscale devices. They are promising candidates for various applications, including filtration, protective clothing, polymer batteries, and sensors. ${ }^{1-4}$ Furthermore, their high surface-to-volume ratio renders them attractive as catalyst supports as well as in drug delivery and tissue engineering. ${ }^{5-8}$ Electrospinning, one of the most established fiber fabrication methods, has attracted much attention due to the ease by which fibers with diameters ranging from $10 \mathrm{~nm}$ to $10 \mu \mathrm{m}$ can be produced from natural or synthetic materials. ${ }^{9-11}$ However, this method requires a dc voltage in the $\mathrm{kV}$ range and high fiber production rates are difficult to achieve because only a single fiber emerges from the nozzle of the pipet holding the polymer solution. ${ }^{12}$

Here, we report a simple but efficient procedure enabling the parallel fabrication of a multitude of polymer fibers with regular morphology and diameters as small as $25 \mathrm{~nm}$. It involves the application of drops of a polymer solution onto a standard spin coater, followed by fast rotation of the chuck, without the need of a mechanical constriction. The fiber formation relies upon the instability of the spin-coated liquid film that arises due to a competition of the centrifugal force and the Laplace force induced by the surface curvature. This Rayleigh-Taylor instability triggers the formation of thin liquid jets emerging from the outward driven polymer

\footnotetext{
*Corresponding author. E-mail: t.weitz@fkf.mpg.de.

${ }^{\dagger}$ Max Planck Institute for Solid State Research.

Max Planck Institute for Metals Research.

\& Institute for Theoretical and Applied Physics, Universität Stuttgart.

"Institut de Physique des Nanostructures, Ecole Polytechnique Fédérale de Lausanne.
}

solution, yielding solid nanofibers after evaporation of the solvent. In addition to being simple, the spinning procedure offers several technologically relevant advantages, including the absence of the need of a mechanical constriction and the ability to yield hollow polymer beads, and is applicable to different types of polymers.

We have focused on the formation of nanofibers made of poly-(methylmethacrylate) (PMMA), which can be regarded as a prototype system for other polymers. In the centrifugal spinning experiments, an aliquot of a PMMA polymer solution was placed in the middle of the chuck of a spincoater, which was then rotated at a speed of at least 3000 rotations per minute (rpm) for a few seconds (Figure S1 Supporting Information). The typical volume and concentration of the applied PMMA solution was $2 \mathrm{~mL}$ and $5 \mathrm{wt} \%$ in chlorobenzene, respectively, with polymer molecular weights of the order of $10^{4} \mathrm{~kg} / \mathrm{mol}$. After spinning, PMMA nanofibers were collected at the edge of the spin-coater. Their diameter was determined by scanning electron microscopy (SEM) to be in the range of $25 \mathrm{~nm}$ to $5 \mu \mathrm{m}$, and fiber lengths of up to $0.5 \mathrm{~mm}$ were detected.

The liquid film created on the rotating chuck expands outward through the enacting centrifugal force in an unsymmetric pattern: we observe the formation of expanding edges and fingers of liquid. Such fingering instabilities are a common phenomenon occurring at the line of contact of a liquid that is forced to spread on a substrate due to external driving forces such as gravitational forces, ${ }^{13}$ capillary forces ${ }^{14}$ centrifugal forces,${ }^{15}$ and Marangoni forces (gradients in surface tension). ${ }^{16-18}$ The liquid flows prefer- 

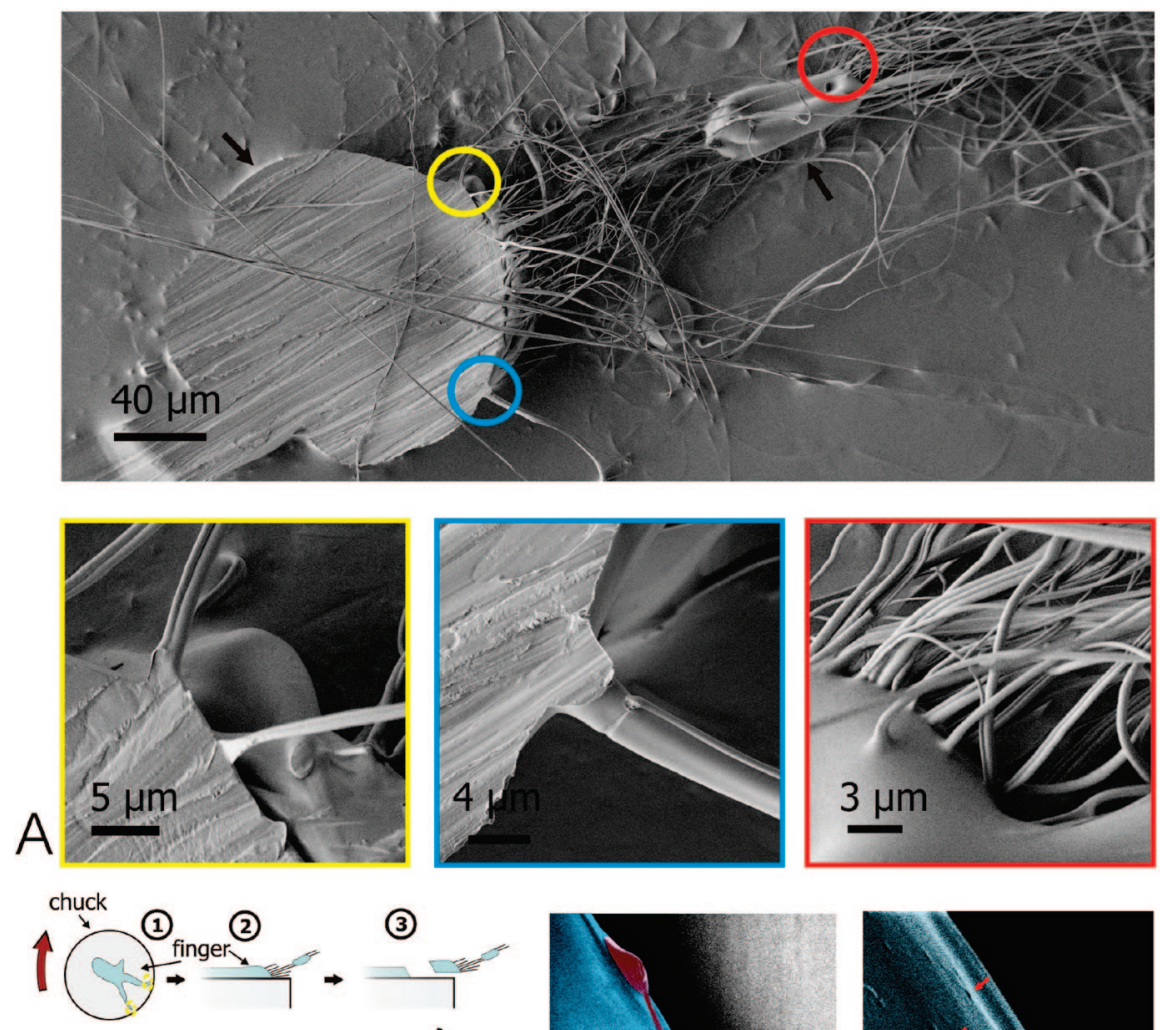

(3)
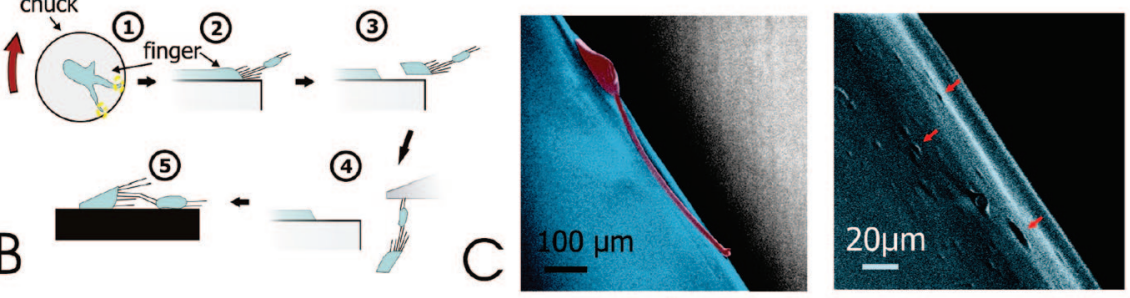

Figure 1. Scanning electron microscopy (SEM) images of the fiber formation. (A) SEM image of a solidified polymer finger (lower left part, marked by an arrow) and a droplet that emerged from this finger (upper right part, marked by an arrow). The droplet is connected via numerous fibers to the finger. The formation of both the droplets and the nanofibers is caused by the Rayleigh-Taylor instability. In this experiment, a $2 \mathrm{~mL}$ drop of a $5 \mathrm{wt} \%$ solution of $950 \mathrm{~kg} / \mathrm{mol}$ PMMA was deposited onto the chuck, which was then rotated at $8000 \mathrm{rpm}$ for $30 \mathrm{~s}$. The three encircled regions, marked by different colors, have been inspected in closer detail. The first region (yellow) reveals a distance between two ejected fibers of approximately $7 \mu \mathrm{m}$. The blue framed region shows a nanofiber emerging from a surface instability, while the red one displays multiple fibers emerging from the same droplet. The distance between these fibers ranges between about 1 and $5 \mu \mathrm{m}$. (B) Schematic depiction of the presumed formation mechanism of fingers and fibers like those shown in panel (A). It involves the following five steps: (1) After deposition in the middle of the chuck, the PMMA solution extends outward upon spinning with concomitant formation of fingers. (2) At the finger ends, droplets and jets of polymer solution are ejected (marked in yellow). At a later stage, the tip of the finger breaks off (3), followed by arrival of the fragment at the edge of the spin coater (4), from where it is deposited onto a substrate (5) for further characterization. (C) SEM image of the edge of a spin-coated PMMA film prepared from of $5 \mathrm{wt} \%$ solution of $950 \mathrm{~kg} / \mathrm{mol}$ PMMA on a silicon wafer (colored in blue) under conditions that avoid the fingering instability (left panel). The surface instability at the edge of the PMMA-coated silicon wafer from which a PMMA nanofiber emerges is highlighted in red. (right panel) SEM micrograph of a larger number of surface instabilities (three are marked by red arrows) on the edge of a PMMA film on a silicon wafer, with a separation on the order of several micrometers.

entially through these fingers, causing them to grow relative to other portions of the expanding pattern. A closer microscopic view of such a finger is presented in Figure 1A; a scheme of the presumable formation of these fingers is shown in Figure 1B. At the end of these fingers nanofibers can be observed (Figure 1A). Their formation can be explained by a Rayleigh-Taylor instability at the air-liquid interface.

Surface instabilities are well-known to be responsible for related phenomena such as droplet formation in the case of dripping of a liquid coating from a ceiling. ${ }^{19-21}$ This dripping occurs because the interface between a liquid film and a gas layer can become unstable to infinitesimal vertical deformations such that, after a while, the liquid film turns into an array of drops. By formulating a rough balance between the destabilizing gravitational force per volume $\rho g \partial h / \partial x$ and the stabilizing surface tension force per volume $\gamma \partial^{3} h / \partial x^{3}$, the wavelength of the instability, i.e., the mean distance between the drops, can be estimated. One finds that the wavelength is controlled by the so-called capillary length $\kappa^{-1}=(\gamma /(\rho g))^{1 / 2},{ }^{19}$ where $\partial / \partial x \sim \kappa$. Here $g$ is the gravitational acceleration, $\rho$ is the density of the liquid, and $\gamma$ is the liquid-gas surface tension. The height of the liquid film hanging under the horizontal surface and the direction parallel to the ceiling are denoted as $h$ and $x$, respectively. The wavelength of the instability that occurs during the spinning of the polymer solution can be determined by an analogous balancing between the destabilizing centrifugal force $\rho \omega^{2} R$ and the stabilizing surface tension force $\gamma \partial^{3} h / \partial r^{3}$, where $\omega$ 


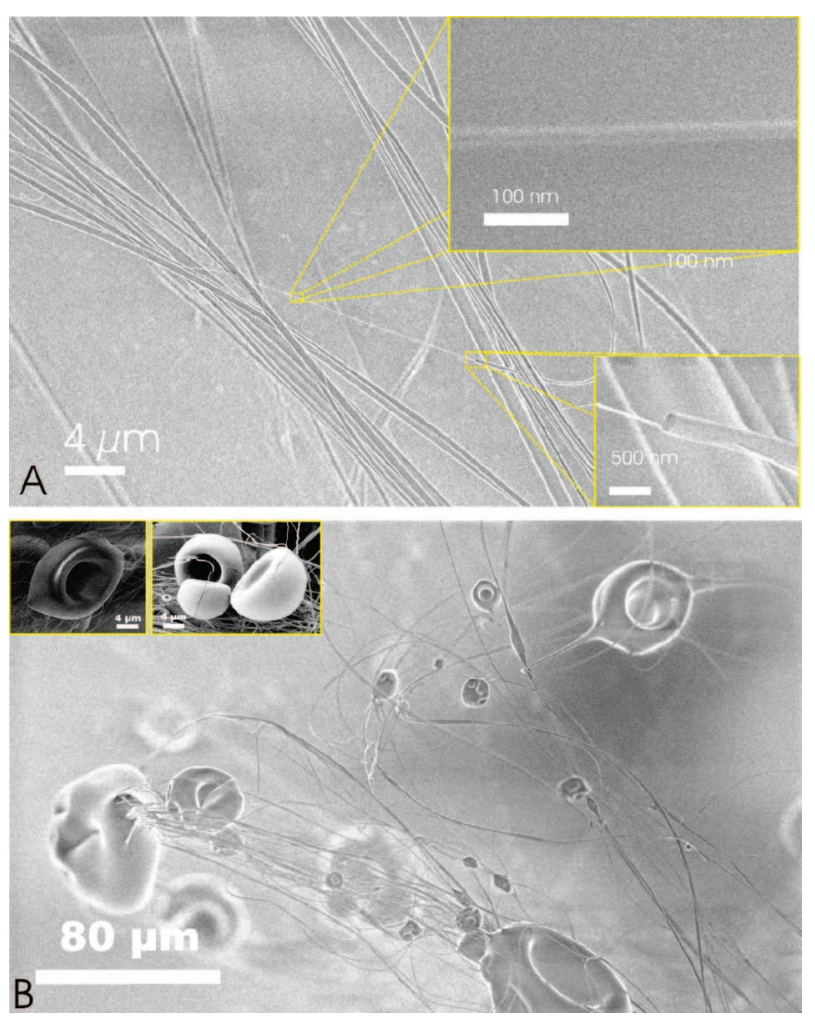

Figure 2. SEM images of nanoscopic polymer fibers and beads. (A) SEM image of PMMA nanofibers obtained from a 5 wt $\%$ chlorobenzene solution of $950 \mathrm{~kg} / \mathrm{mol}$ PMMA subjected to a rotation speed of $3000 \mathrm{rpm}$. The diameter of the fiber shown in the upper inset is $25 \mathrm{~nm}$. As apparent from the lower inset, the fibers are massive. (B) SEM image of nanofibers that connect polymer beads of various sizes, obtained by spinning of a 5 wt \% chlorobenzene solution of $950 \mathrm{~kg} / \mathrm{mol}$ PMMA at $3000 \mathrm{rpm}$. Insets: Magnified views of torodial and cuplike structures.

is the angular speed, $R=0.0325 \mathrm{~m}$ is the radius of the chuck, and $r$ is the distance of the polymer solution from the center of the chuck in cylindrical coordinates. The characteristic length scale of the instability is then given by $L=$ $\left(h \gamma /\left(\rho \omega^{2} R\right)\right)^{1 / 3}$, where $\partial / \partial r \sim L^{-1}$. Using the parameters $\omega$ $=500 \mathrm{~s}^{-1}, \gamma=0.01 \mathrm{~kg} / \mathrm{s}^{2}, \rho=10^{3} \mathrm{~kg} / \mathrm{m}^{3}$, and $h=0.1 \mu \mathrm{m}$, one obtains $L=4 \mu \mathrm{m}$ for the wavelength of the instability. This value is in good agreement with the mean distance between the points of ejection of the polymer fibers at the end of the fingers (Figure 1A). The surface instabilities are the regions where liquid jets emerge due to the high radial speed $v=\omega R$.

The reason why the ejected jets of polymer solution do not further break up into individual droplets, but rather give rise to continuous, solid nanofibers (Figure 2), is the relatively high viscosity of the utilized PMMA solutions (of the order of several $10 \mathrm{mPa} \cdot \mathrm{s})$. Stretching of the fibers by the centrifugal force exerted onto the polymer and evaporation of the solvent molecules during the movement toward the collecting area is expected to cause thinning of the fibers. The interplay between the centrifugal force and the viscous properties of the polymer solution is the reason that the spincoating method is able to produce nanofibers, whereas conventional mechanical fiber spinning techniques, in which the diameter of the produced wires is defined by a mechanical constriction, yield fibers with diameters of at least a few tens of micrometers.

It is interesting to note that polymer nanofibers could be obtained even when the formation of fingers was suppressed either by depositing an increased amount of polymer solution on the chuck or via deposition of the solution close to the edge of the chuck. To clarify the fiber formation mechanism in these cases, a silicon wafer substrate was fixed onto the chuck and a drop of PMMA solution placed close to the edge of the wafer. Scanning electron micrographs revealed that the resulting fibers originate from surface instabilities that occur when the polymer solution spreads across the wafer edge, as exemplified in the left panel of Figure 1C. Further surface instabilities of the polymer film at the edge of the waver are shown in Figure 1C in the right panel.

The fiber formation mechanism was further elucidated by investigating the influence of the concentration and molecular weight of the PMMA, as well as the spinning speed of the chuck. The data collected in Table 1 indicate under which conditions PMMA nanofibers could be observed. It is apparent that nanofiber formation requires that the polymer concentration exceeds the so-called entanglement concentration, which is about $3 c^{*}$, where $c^{*}$ is the overlap concentration. This finding corresponds well with a recent study on electrospinning of PMMA solution ${ }^{22}$ and can be attributed to the fact that in both the electrospinning and the present spin-coating method, uniform fibers are difficult to obtain due to the insufficient chain overlap when the polymer solution is too dilute.

It can be furthermore seen that the minimum rotation speed needed for fiber formation is found to increase with decreasing viscosity of the polymer solution. This dependency can be understood on the basis of the viscoelasticity of the PMMA solutions. Viscoelasticity is a property common to polymer solutions and accounts for the observation that both the viscosity and the elasticity of the polymer solution depend on the strength and time constant of external forces acting on the polymer chains. Specifically, if the time constant of the external force is small, the response of the polymer solution is elastic, whereas long time constants result in a viscous response of the polymer solution. Hence, for spinning of low viscosity polymer solutions, fibers can be obtained provided the rotation speed is large enough to increase the viscosity. In fact, we found that for the $2.5 \mathrm{wt} \%$ solution of $950 \mathrm{~kg} / \mathrm{mol}$ PMMA with a viscosity $\eta=13 \mathrm{mPa} \cdot \mathrm{s}$, fiber generation requires a minimum spinning speed of $8000 \mathrm{rpm}$, whereas in the case of the $5 \mathrm{wt} \%$ solution of $950 \mathrm{~kg} / \mathrm{mol}$ PMMA with viscosity $\eta=57 \mathrm{mPa} \cdot \mathrm{s}$, a speed of $3000 \mathrm{rpm}$ is sufficient. Interestingly, we did not observe a dependence of the minimum fiber diameter on either the concentration of polymer solution or the molecular weight of the PMMA macromolecules.

The morphology of the PMMA fibers was found to sensitively depend on the vapor pressure of the solvent used. In particular, solvents with lower vapor pressure (e.g., ethylactate with a vapor pressure of $2 \mathrm{hPa}$ at $20^{\circ} \mathrm{C}$ instead of chlorobenzene with a vapor pressure of $21 \mathrm{hPa}$ at $20^{\circ} \mathrm{C}$ ) 
Table 1. Collection of the Parameters Used in the Study of Nanofiber Formation as a Function of Molecular Weight, Concentration of the PMMA-Chlorobenzene Solution, and the Rotation Speed ${ }^{a}$

\begin{tabular}{lcccc}
\hline molecular weight & concentration [wt\%] & $c / c^{*}$ & $\eta[\mathrm{mPa} \cdot \mathrm{s}]$ & rpm range when fibers were observed \\
\hline \multirow{2}{*}{$200 \mathrm{~kg} / \mathrm{mol}$} & 3.5 & 1.6 & 4 & \\
& 5.0 & 2.3 & 6 & $8000 \mathrm{rpm}\left(r_{\min }=35 \mathrm{~nm}\right)^{b}$ \\
$950 \mathrm{~kg} / \mathrm{mol}$ & 7.0 & 3.2 & 11 & 6 \\
& 1.5 & 2.0 & 6 & \\
& 2.0 & 2.6 & 7 & $8000 \mathrm{rpm}\left(r_{\min }=45 \mathrm{~nm}\right)^{b}$ \\
& 2.5 & 3.3 & 13 & $3000 \mathrm{rpm}\left(r_{\min }=25 \mathrm{~nm}\right)^{b}$ to $8000 \mathrm{rpm}\left(r_{\min }=50 \mathrm{~nm}\right)^{b}$
\end{tabular}

${ }^{a}$ The rotation speed was increased from 1000 to 8000 rotations per min (rpm) by increments of $1000 \mathrm{rpm}$. The ratio $c / c^{*}$ denotes the ratio of the actual concentration to the chain overlap concentration $c^{*}$ of PMMA. The concentration of polymer solutions can be divided into three regimes: dilute $\left(c / c^{*}<1\right)$, semidilute unentangled $\left(1<c / c^{*}<3\right)$, and semidilute entangled $\left(c / c^{*}>3\right)$. All polymer solutions were purchased from ALLRESIST GmbH, Strausberg, Germany. The last column indicates in which spin speed range nanofibers were observed. ${ }^{b}$ The minimum fiber diameter observed at the respective rotation speed is given in brackets.

resulted in quite thick (>500 $\mathrm{nm}$ diameter) fibers that frequently coalesce and that are much more prone to breaking (see Figure S2, Supporting Information). This difference most likely arises from the easier relaxation of the polymer chains to their nonstretched conformation within a solvent of lower vapor pressure. This lower vapor pressure ensures a slow drying of the fibers.

Finally, it is noteworthy that under all investigated conditions, polymer beads were found as a byproduct of the spinning process. Examples of such toroidal and cuplike beads are shown in Figure 2B. The formation of beads and beaded fibers is driven by surface tension and is another manifestation of the Rayleigh-Taylor instability. Surface tension acts to decrease the surface area per unit mass by changing the jets into spheres. A driven jet of a low molecular weight liquid will form spherical droplets, while viscoelastic forces in polymer solutions resist rapid changes in shape leading to beaded fibers (see, e.g., ref 23). The reason for this is that the coiled macromolecules of the dissolved polymer are transformed by the elongational flow of the jet into oriented, entangled networks that persist as the fiber solidifies. The contraction of the radius of the jet, which is driven by surface tension, causes the remaining solution to form beads. Contrary to electrospinning, in which only one fiber is connected to each bead, in the spinning process, multiple fibers usually emerge from the beads (Figure 2B). The bead diameter increases as the viscosity increases (Figure 3), as is expected for the break-up of a liquid jet due to the Rayleigh-Taylor instability. ${ }^{24}$ For example, the maximum droplet size observed for a $5 \mathrm{wt} \%$ solution of $950 \mathrm{~kg} / \mathrm{mol}$ PMMA with viscosity $\eta=57 \mathrm{mPa} \cdot \mathrm{s}$ was determined to be $90 \mu \mathrm{m}$, whereas the maximum droplet size is below $30 \mu \mathrm{m}$ when a $2.5 \mathrm{wt} \%$ solution of $950 \mathrm{~kg} /$ mol PMMA with viscosity $\eta=13 \mathrm{mPa}$.s is used. The obtained beads are potentially of interest for use in controlled drug release or as protective layers on fabrics. ${ }^{25-27}$

As a first step toward expanding the application range of the spinning method, we have successfully obtained nanofibers and beads from a (methylmethacrylate-methacrylic acid)-based copolymer, which is more hydrophilic compared to PMMA. Examples of such fibers are depicted in Figure S3 in the Supporting Information. By careful optimization of the relevant parameters, such as viscosity of the polymer solution, spinning speed, surface tension, and
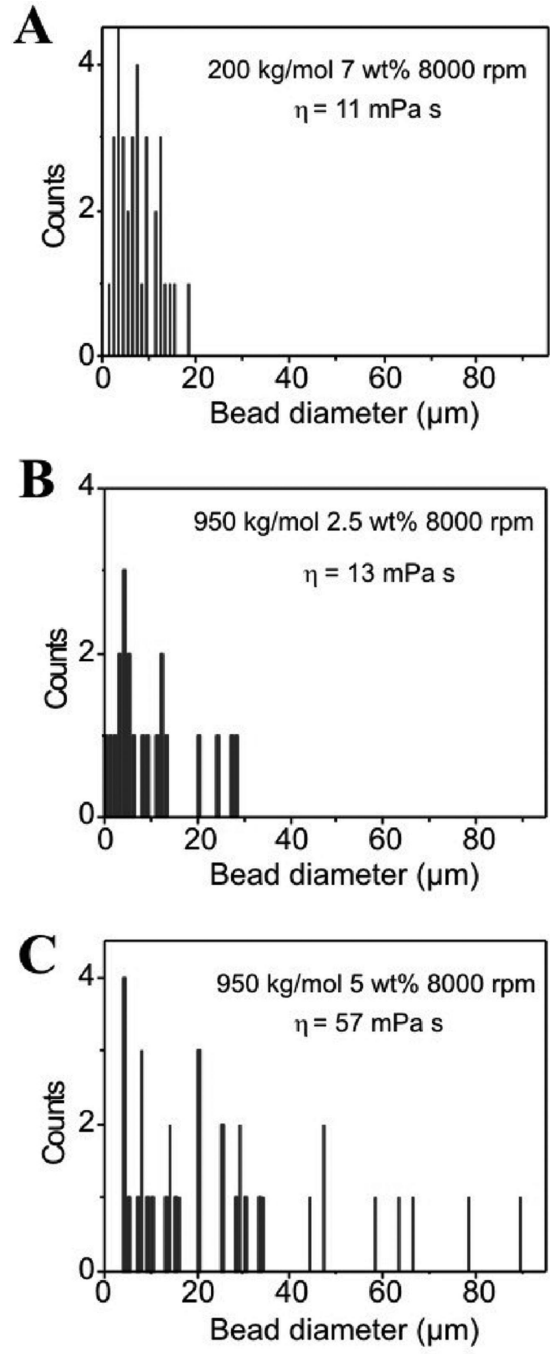

Figure 3. Statistics of the measured bead diameters in dependence of the viscosity of the polymer solution used for spin-coating. With increasing viscosity from (A) to (C), the average diameter of the beads increases, in agreement with the Rayleigh-Taylor instability that is responsible for the formation of the beads.

solvent evaporation rates, the method could be extended to further types of polymers, including materials not amenable to electrospinning.

Supporting Information Available: Schematic illustration of the spinning procedure used for the fabrication of 
polymer nanofibers. SEM images of PMMA polymer fibers fabricated using ethylactate as solvent. SEM image of fibers obtained when a copolymer is used for the fabrication. This material is available free of charge via the Internet at http:// pubs.acs.org.

Acknowledgment. We acknowledge stimulating discussions with Angel Rubio.

\section{References}

(1) Ramakrishna, S.; Fujihara, K.; Teo, W.-E.; Lim, T.-C.; Ma, Z., An Introduction to Electrospinning and Nanofibers; World Scientific: Singapore, 2005.

(2) Dersch, R.; Steinhart, M.; Boudriot, U.; Greiner, A.; Wendorff, J. H. Polym. Adv. Technol. 2005, 16 (2-3), 276-282.

(3) Deitzel, J. M.; Kleinmeyer, J.; Harris, D.; Tan, N. C. B. Polymer 2001, 42 (1), 261-272.

(4) Ko, F.; Gogotsi, Y.; Ali, A.; Naguib, N.; Ye, H. H.; Yang, G. L.; Li, C.; Willis, P. Adv. Mater. 2003, 15 (14), 1161-1165.

(5) Stevens, M. M.; George, J. H. Science 2005, 310 (5751), 1135-1138.

(6) Pham, Q. P.; Sharma, U.; Mikos, A. G. Tissue Eng. 2006, 12 (5), 1197-1211.

(7) Li, W. J.; Laurencin, C. T.; Caterson, E. J.; Tuan, R. S.; Ko, F. K. J. Biomed. Mater. Res. 2002, 60 (4), 613-621.

(8) Matthews, J. A.; Wnek, G. E.; Simpson, D. G.; Bowlin, G. L. Biomacromolecules 2002, 3 (2), 232-238.

(9) Dzenis, Y. Science 2004, 304 (5679), 1917-1919.

(10) Reneker, D. H.; Chun, I. Nanotechnology 1996, 7 (3), 216-223.

(11) Bognitzki, M.; Czado, W.; Frese, T.; Schaper, A.; Hellwig, M.;
Steinhart, M.; Greiner, A.; Wendorff, J. H. Adv. Mater. 2001, 13 (1), 70-72.

(12) Greiner, A.; Wendorff, J. H. Angew. Chem., Int. Ed. 2007, 46 (30), 5670-5703.

(13) Huppert, H. E. Nature 1982, 300 (5891), 427-429.

(14) Troian, S. M.; Wu, X. L.; Safran, S. A. Phys. Rev. Lett. 1989, 62 (13), 1496-1499.

(15) Melo, F.; Joanny, J. F.; Fauve, S. Phys. Rev. Lett. 1989, 63 (18), 19581961.

(16) Scriven, L. E.; Sternling, C. V. Nature 1960, 187 (4733), 186-188.

(17) Cazabat, A. M.; Heslot, F.; Troian, S. M.; Carles, P. Nature 1990, 346 (6287), 824-826.

(18) Sur, J.; Witelski, T. P.; Behringer, R. P. Phys. Rev. Lett. 2004, 93 (24), 247803.

(19) de Gennes, P.-G.; Brochard-Wyart, F.; Quere, D., Capillarity and Wetting Phenomena; Springer: New York, 2004.

(20) Burgess, J. M.; Juel, A.; McCormick, W. D.; Swift, J. B.; Swinney, H. L. Phys. Rev. Lett. 2001, 86 (7), 1203-1206.

(21) Suryo, R.; Basaran, O. A. Phys. Rev. Lett. 2006, 96 (3), 034504.

(22) Gupta, P.; Elkins, C.; Long, T. E.; Wilkes, G. L. Polymer 2005, 46 (13), 4799-4810.

(23) Fong, H.; Chun, I.; Reneker, D. H. Polymer 1999, 40 (16), 45854592.

(24) Weber, C. Z. Angew. Math. Mech. 1931, 11, 136-154.

(25) Liu, J.; Kumar, S. Polymer 2005, 46 (10), 3211-3214.

(26) Deotare, P. B.; Kameoka, J. Nanotechnology 200617 (5), 13801383.

(27) Eda, G.; Shivkumar, S. J. Mater. Sci. 2006, 41 (17), 5704-5708. NL080124Q 